\title{
Explicit Inverses and Condition Numbers of Certain Girculants
}

\author{
By S. Charmonman and R. S. Julius
}

\begin{abstract}
Explicit inverses and condition numbers of two test-circulants with first rows $\{a, a+h, \cdots, a+(n-1) h\}$ and $\left\{a, a h, \cdots, a h^{n-1}\right\}$ respectively are given in terms of the parameters defining the circulants.
\end{abstract}

1. Introduction. A circulant with the first row $(1,2, \cdots, n)$ was used in testing inversion algorithms [6] but its explicit inverse was not given in the list of explicit inverses of some particular matrices in [5]. This paper gives explicit inverses and condition numbers of two circulants whose special cases include the one used in [6].

The simple result that the inverse of a circulant is a circulant [2] can be easily extended to the case of $r$-circulants. An $r$-circulant as defined in [4] is a square matrix of order $n$ in which the $i$ th row, $i=2,3, \cdots, n$, is obtained from the $(i-1)$ th row by cyclically shifting each element $r$ places to the right. The word "row" can be replaced by the word "column" if "right" is replaced by "down." If we also say that shifting a negative number of places right means shifting left, then an $r$-circulant is also a $(k n+r)$-circulant, for any integer $k$.

Theorem 1.1. The inverse of a nonsingular r-circulant $A$ is an s-circulant $B$ where s satisfies

$$
r s=k n+1
$$

for some integer $k$.

Proof. Let $e_{1}$ denote the first column of $I$. The set of equations

$$
A f=e_{1}
$$

has a unique solution $f$, since $A$ is nonsingular. Let $B$ be the $s$-circulant with first column $f$, where $s$ satisfies (1.1). Then, by Theorem (3.1) in [1], $A B$ is an $(r s=k n+1)$ circulant with first column $e_{1}$; that is, $A B=I$.

2. Explicit Inverses. Since the inverse of a circulant is a circulant we shall hypothesize that the inverse of a circulant which is defined by a few parameters can be explicitly expressed in terms of these parameters. The forms of the expressions can be conveniently observed from the results of numerical experiments on a digital computer and applications of (1.2) then give the required explicit inverses.

In the following two theorems $s$ is defined as in (1.1). $A_{1}$ and $A_{2}$ are nonsingular $r$-circulants of order $n \geqq 2$ with first row $\{a, a+h, \cdots, l=a+(n-1) h\}$ for $A_{1}$, and $\left\{a, a h, \cdots, a h^{n-1}\right\}$ for $A_{2} . R_{i}(A)$ denotes the $i$ th row, $C_{i}(A)$ the $i$ th column and $a_{i j}$ the $(i, j)$ th element of $A$.

Received May 18, 1967. Revised August 28, 1967. 
Theorem 2.1. The inverse of $A_{1}$ is the s-circulant with the first column $\{b-\alpha, b, \cdots, b, b+\alpha\}^{T}$, where $b=2 /\left\{n^{2}(a+l)\right\}$ and $\alpha=1 /(n h)$.

Proof. Let $y$ be the first column of an $s$-circulant $B$. According to (1.2) $B$ will be the inverse of $A$ if the $i$ th element of

$$
A_{1} y=R_{i}(A) C_{1}(B)=b \sum_{i=1}^{n}[a+(i-1) h]+\alpha\left(a_{i n}-a_{i 1}\right)
$$

is $e_{1}$. By observation of $A_{1}$ we have

$$
\begin{aligned}
a_{\text {in }}-a_{i 1} & =(n-1) h, & & i=1, \\
& =-h, & & i \neq 1 .
\end{aligned}
$$

Therefore, $A_{1} y=e_{1}$ if $\alpha n h=1$ and $b n(a+l) / 2-\alpha h=0$ which give the required expressions for $b$ and $\alpha$.

Obviously Theorem 2.1 gives the inverse of a $( \pm 1)$-circulant with first row $(1,2, \cdots, n)$ as the $( \pm 1)$-circulant with the first column $n^{-1}(b-1, b, \cdots, b, b+1)^{T}$ where $b=2 /\{n(n+1)\}$.

THEOREM 2.2. The inverse of $A_{2}$ is the s-circulant with first column $\{b, 0, \cdots, 0,-h b\}^{T}$ when $b=1 /\left\{a\left(1-h^{n}\right)\right\}$.

A proof can be easily constructed similar to that for Theorem 2.1 by using the property of $\mathrm{A}_{2}$ that

$$
\begin{aligned}
h a_{\text {in }}-a_{i 1} & =a\left(h^{n}-1\right), & & i=1, \\
& =0, & & i \neq 1 .
\end{aligned}
$$

3. Condition Numbers. Circulants which are usually employed in testing numerical algorithms are the 1-circulant which are generally nonsymmetric and the $(-1)$-circulants which are always symmetric. One measure of the condition of these matrices, denoted here by $P(A)$, is the ratio of the largest (in modulus) to the smallest eigenvalue [7].

An expression for the eigenvalues of a 1-circulant may be found in [3] and [5]:

$$
\lambda_{s}=\sum_{j=1}^{n} a_{j} t_{s}^{j-1}
$$

where $t_{s}=\cos (2 \pi s / n)+i \sin (2 \pi s / n), s=1,2, \cdots, n$, and $a_{j}$ is the $j$ th element of the first row of the circulant.

An expression for the $n$ eigenvalues of a $(-1)$-circulant does not seem to be readily available in the literature but can be easily shown to be

$$
\lambda_{0} ; \pm\left(\lambda_{s} \lambda_{n-8}\right)^{1 / 2}, \quad s=1,2, \cdots,(n-1) / 2,
$$

for odd $n$. The $\lambda_{s}$ are the eigenvalues of the 1-circulant whose first row is the same as the $(-1)$-circulant in question. When $n$ is even the set of $n$ eigenvalues of the $(-1)$-circulant are $\lambda_{(n / 2)}$ and those in (3.3) with $s=1,2, \cdots,(n-2) / 2$.

Examinations of the sets of eigenvalues of the $( \pm 1)$-circulants of the forms $A_{1}$ and $A_{2}$ did not reveal expressions for their condition numbers. Since the explicit inverses of $A_{1}$ and $A_{2}$ as given in Theorems 2.1 and 2.2 are simpler than the matrices themselves, eigenvalues of the inverses were examined. After algebraic and 
trigonometric manipulations, we have for both the 1-circulants and (-1)-circulants,

$$
P\left(A_{1}\right) \sim n+2 a / h, \quad h>0
$$

and

$$
P\left(A_{2}\right) \sim \operatorname{Max}\left[p_{2}=|1+h| /|1-h|, 1 / p_{2}\right],
$$

for large $n$. The symbol " " is read "asymptotically equals." In the special case in which $a=h=1$ in $A_{1}$, (3.4) gives $P\left(A_{1}\right) \sim n$ in agreement with the result in [5].

4. Acknowledgement. The preparation of this paper was supported by the National Research Council of Canada Grant No. NRC A-4076.

Department of Computing Science,

University of Alberta,

Edmonton, Alberta, Canada.

1. C. M. Ablow \& J. L. Brenner, "Roots and canonical forms for circulant matrices," Trans, Amer. Math. Soc., v. 107, 1963, pp. 360-376. MR 27 \#5775.

2. A. C. AItKen, Determinants and Matrices, Oliver and Boyd, Edinburgh, 1939; Interscience, New York, 1956, pp. 123-124. MR 1, 35.

3. Richard Bellman, Introduction to Matrix Analysis, McGraw-Hill, New York, 1960, pp. 234-239. MR 23 \#A153.

4. B. Friedman, "Eigenvalues of composite matrices," Proc. Cambridge Philos. Soc., v. 57, 1961, pp. 37-49. MR 23 \#A159.

5. Marvin Marcus, "Basic theorems in matrix theory," Nat. Bur. Standards Appl. Math. Ser., v. 57, 1960, p. 9, pp. 21-23. MR $22 \# 709$.

6. Morris Newman \& John Todd, "The evaluation of matrix inversion programs," J. Soc. Indust. Appl. Math., v. 6, 1958, pp. 466-476. MR $20 \# 6781$.

7. JoHn TodD, "The condition of a certain matrix," Proc. Cambridge Philos. Soc., v. 46, 1950, pp. 116-118. MR 11, 403. 